\title{
STRUCTURE FUNCTION ANALYSIS APPLIED TO THE METSÄHOVI SAMPLE
}

\author{
MARKKU LAINELA \\ Tuorla Observatory, University of Turku \\ SF-21500 Piikkiö, Finland \\ and \\ ESKO VALTAOJA \\ Metsähovi Radio Research Station \\ Metsähovintie \\ SF-02540 Kylmälä, Finland
}

Our monitoring program in Metsähovi, Finland, consists of a complete sample of bright, flat-spectrum extragalactic compact radio sources. For the structure function analysis we have selected 42 best observed objects from this sample. The structure function analysis gives us two parameters, the slope value $\alpha$ and the maximum variability timescale $T_{m a x}$.

In our sample the slope value a varies between 0.6 and 3.9 , but in most cases $\alpha \leq 1.5$. The mean value for $\alpha$ is 1.2 at $22 \mathrm{GHz}$ and 1.3 at $37 \mathrm{GHz}$. All sources with $\alpha>1.5$ have flux curves that are dominated by one or a few prominent outbursts that steepen the structure function. It is likely that over a greater timespan even these sources would have structure functions with smaller slopes. Thus, one can say as a general conclusion that in most, if not all, cases the typical noise type at 22 and $37 \mathrm{GHz}$ is shot noise (i.e., $\alpha \approx 1$ ). This is consistent with there being one basic physical mechanism of millimeter variability for all compact radio sources and together with the successfully modelling at centimeter wavelengths our results support the idea that shock-in-jet models can explain most of the time variability in all compact radio sources.

The time scales of variability support an orientation-dependent model, where HPQs have smaller average viewing angles than LPQs. In many scenarios one assumes that in BL Lacs the jet is pointing almost directly towards us and so $T_{\max }$ for BL Lacs should be the smallest among AGN. However, we do not find this kind effect in our data, on the contrary there seems to be a wide spread in $T_{m a x}$. One interesting possibility is that BL Lacs really consist of two different classes of objects (Valtaoja et al. 1992).

\section{References}

Lainela, M., \& Valtaoja, E. 1993, ApJ (in press)

Valtaoja, E., Teräsranta, H., Lainela, M., \& Teerikorpi, P. 1992, in Variability of Blazars, ed. E. Valtaoja \& M. Valtonen (Cambridge: Cambridge Univ. Press), 70

396

T. J.-L. Courvoisier and A. Blecha: Multi-Wavelength Continuum Emission of AGN, 396.

(C) 1994 IAU. Printed in the Netherlands. 